Revista Brasileira de Agricultura Irrigada v.10, nº.1, p. 449 - 459, 2016

ISSN 1982-7679 (On-line)

Fortaleza, CE, INOVAGRI - http://www.inovagri.org.br

DOI: $10.7127 /$ rbai.v10n100386

Protocolo 386.16 - 04/11/2015 Aprovado em 16/01/2016

\title{
PRODUÇÃO DE MUDAS DE PIMENTÃO IRRIGADAS COM EFLUENTES DE PISCICULTURA
}

Thiara Silvestre Nascimento ${ }^{1}$, Luciane Sperandio Floriano ${ }^{2}$, Alexandre Igor de Azevedo Pereira $^{3}$, Raimundo Nonato Farias Monteiro ${ }^{4}$, Mariana Alexandre de Lima Sales ${ }^{5}$.

\section{RESUMO}

O uso de águas residuárias na agricultura é uma alternativa rentável, pois contribui com o desenvolvimento sustentável e o reuso de nutrientes essenciais. A cultura do pimentão, uma cultura bastante utilizada em todo mundo, principalmente no Brasil, é uma cultura em que se utiliza de grandes quantidades de insumos químicos, sendo assim, o uso de água residuária, vem para melhor sua produção. Este ensaio teve por objetivo avaliar o desenvolvimento de mudas de pimentão (Cascadura Ikeda) irrigadas com diferentes concentrações de efluente de piscicultura. O trabalho foi realizado Instituto Federal Goiano - Campus de Urutaí - GO. O delineamento estatístico adotado foi inteiramente casualizado com 16 repetições, às quais foram ministradas seis níveis de concentração de água residuária na irrigação. A água de irrigação estava com porcentagem de água residuária 0, 25, 50, 75 e 100\%. As variáveis analisadas foram: altura das plantas e largura das folhas aos 5, 10, 15, 20, 25, 30, 35, 40, 45 e 50 dias após a germinação, número de folhas verdadeiras, peso de matéria fresca da parte aérea, peso de matéria fresca do sistema radicular, peso de matéria seca da parte aérea e peso de matéria seca do sistema radicular. De posse dos resultados verifica-se que, a elevação das concentrações de água residuária de piscicultura, apresentou aumento nos valores das variáveis analisadas, podendo concluir que, a água residuária da piscicultura apresenta-se como alternativa para reuso do ponto de vista nutricional para as plantas, diminuindo o impacto ambiental negativo dos efluentes desse tipo de criação animal.

Palavras-chave: Capsicum annuum L., água residuária, concentrações, irrigação.

\section{PRODUCTION OF PEPPER SEEDLINGS IRRIGATED WITH EFFLUENT OF PISCICULTURE}

\footnotetext{
ABSTRACT

1 Tecnóloga em Irrigação e Drenagem, Mestranda em Agronomia (Irrigação e Drenagem), UNESP/FCA, Botucatu - SP, e-mail: thiarasilvestre@hotmail.com.

${ }^{2}$ Zootecnista, Dra. em Ciência Animal, Profa. IFGoiano, Câmpus de Urutaí - GO.

${ }^{3}$ Eng. Agrônomo, Dr. em Entomologia, Prof. IFGoiano, Câmpus de Urutaí - GO.

${ }^{4}$ Tecnólogo em Rec. Hídricos/Irrigação, Dr. em Agronomia (Irrigação e Drenagem), UNESP/FCA, Botucatu SP.

${ }^{5}$ Tecnóloga em Irrigação e Drenagem, Doutoranda em Agronomia (Irrigação e Drenagem), UNESP/FCA, Botucatu - SP.
} 
The use of residual water in agriculture is a profitable alternative, because it contributes to sustainable development and the reuse of essential nutrients. The pepper culture, a culture widely used worldwide, primarily in Brazil, is a culture which uses large amounts of chemical products, thus the use of wastewater, is best for their production. This trial aimed to evaluate the development of pepper seedlings (Cascadura Ikeda) irrigated with different fish farm effluent concentrations. The work was carried Instituto Federal Goiano - Campus of Urutaí Goias State, Brazil. The statistical design was completely randomized with 16 repetitions, which will be taught six levels of concentration of wastewater for irrigation. The irrigation water will percentage of wastewater with $0,25,50,75$ and $100 \%$. The variables analyzed were: plant height and width of the leaves at 5, 10, 15, 20, 25, 30, 35, 40, 45 and 50 days after germination, number of true leaves, weight of fresh matter of shoots, weight of fresh weight of roots, dry weight of shoot and dry weight of the root system. With the results it appears that the increase in wastewater concentrations of fish farming, increased the values of the variables, which may conclude that the wastewater from fish farming is presented as an alternative to reuse the nutritional point of view for plants, reducing the environmental impact of effluents such animal husbandry.

Keywords: Capsicum annuum L., wastewater, concentrations, irrigation.

\section{INTRODUÇÃO}

O pimentão (Capsicum annuum L.) vem se destacando como uma das olerícolas mais consumidas no Brasil. A sua produção cresceu vertiginosamente nos últimos anos, em função da melhor adaptação em ambiente protegido comparado a outras culturas (LEONARDO et al., 2007). É pertencente à família das Solanáceas, gênero Capsicum, e representa uma das dez hortaliças mais importantes do mercado brasileiro, sendo os frutos consumidos verdes ou maduros. O cultivo do pimentão é de retorno rápido, por isso é largamente explorada por pequenos e médios horticultores (FILGUEIRA, 2000).

Embora muito cultivado por pequenos e médios produtores brasileiros, o pimentão necessita de uso de tecnologia mais adequada nos aspectos relacionados ao manejo, adubação, variedades e tecnologia de irrigação para que sua exploração seja tecnicamente conduzida e economicamente viável, já que esta é uma cultura na qual têm-se utilizado grandes quantidades de insumos químicos (FILGUEIRA, 2004).

A utilização de efluentes na agricultura cresceu consideravelmente nos últimos anos em muitos países, inclusive no Brasil; no entanto, ainda não foram suficientemente estudados todos os aspectos positivos e negativos dessa técnica, especialmente sobre as propriedades físicas e químicas do solo, absorção de nutrientes pelas plantas ou sua toxidez. Dentre os principais fatores que vieram a contribuir para que, nos últimos anos, aumentasse o interesse pela irrigação com efluentes, estão a escassez de recursos hídricos, o avanço do conhecimento técnico-científico, a legislação ambiental mais rigorosa e atuante, o maior controle da poluição ambiental, com redução de problemas à saúde humana e animal, a diminuição dos custos de tratamento devido à atuação do solo como forma de disposição e fornecimento de nutrientes e matéria orgânica às plantas, reduzindo os custos com fertilizantes químicos comerciais (SANDRI, 2003).

O aproveitamento de efluentes de viveiros de peixes para a irrigação e possível complemento de adubação, sendo usado como uma associação rentável tanto econômica como ambiental. A utilização de efluentes de viveiros de peixes reduz o custo da água e a quantidade de fertilizantes minerais sintéticos utilizados (SARIG, 1994).

Desta forma, objetivou-se avaliar o desenvolvimento de mudas de pimentão (Cascadura Ikeda) irrigadas com diferentes concentrações de efluente de piscicultura. 


\section{MATERIAL E MÉTODOS}

A pesquisa foi conduzida nas dependências do Instituto Federal Goiano Câmpus de Urutaí - GO, apresentando latitude de $17^{\circ} 27^{\prime} 52^{\prime \prime}$ Sul e 48 $12^{\prime} 13^{\prime \prime}$ Oeste de longitude e com altitude média de $821 \mathrm{~m}$. O clima da região segundo classificação Köppen é caracterizado como Cwa, sendo representado como clima úmido tropical, de inverno seco e verão chuvoso.

As mudas de pimentão (Cascadura Ikeda) foram implantadas em bandeja com 128 cédulas preenchidas com o substrato Bioplant ${ }^{\circledR}$, que tem como matéria prima casca de Pinus e como agregantes, fibra de coco, casca de arroz e nutrientes, possuindo $\mathrm{pH}$ entre 5,5 - 6,0. O substrato foi peneirado com auxílio de uma peneira com malha de $2 \mathrm{~mm}$ e em seguida colocado na bandeja. Em cada cédula foi inserida uma semente de pimentão, com profundidade de $0,5 \mathrm{~cm}$, e em seguida uma nova camada de substrato foi reposta para a cobertura das sementes, concluindo assim o processo de semeadura.

A água utilizada na irrigação das mudas era oriunda de um poço de decantação, situado no setor de Piscicultura do IFGoiano, Câmpus de Urutaí, e transportada em recipientes para casa de vegetação, de onde eram preparadas as concentrações de 0, 25, 50, 75, 100 e 125\% da água residuária em água potável, que seriam aplicadas nas mudas. Foram encaminhas uma amostra de cada concentração para o laboratório de solos, também localizado no IFGoiano, Câmpus de Urutaí para as devidas análises de macro (P, K, Ca) e micronutrientes (Fe, Mn, $\mathrm{Cu}, \mathrm{Na}, \mathrm{Cu}, \mathrm{Zn}$,)
O delineamento experimental foi inteiramente casualizado. As bandejas foram divididas em 6 tratamentos, sendo seis concentrações de água residuária de piscicultura e 16 repetições.

Foram analisadas a altura das plantas (AP) e largura das folhas (LF) aos 5, 10, 15, 20, 25, 30, 35, 40, 45 e 50 dias após a germinação (DAG), com o auxílio de uma trena milimetrada, bem como número de folhas (NF).

Após atingir o tamanho desejado para o transplantio, as mudas de pimentão foram avaliadas em peso de matéria fresca da parte aérea (PMFPA) e do sistema radicular (PMFSR), ambas medidas em grama, aferidas com uso de uma balança de precisão de $\pm 0,0001 \mathrm{~g}$. Em seguida foram alocadas em envelopes e colocadas em estufa a $70^{\circ} \mathrm{C}$ por 24 horas para então serem feitas as avaliações de peso de matéria seca da parte aérea (PMSPA) e do sistema radicular (PMSSR).

Os resultados foram submetidos à análise de variância e as médias analisadas pelo teste de Tukey a 5\% de probabilidade, com auxílio do software SISVAR, versão 5.3

\section{RESULTADOS E DISCUSSÃO}

Os resultados da análise de macro e micronutrientes para água utilizada na irrigação das mudas de pimentão estão apresentadas na tabela 1. Nota-se que para os micronutrientes cobre $(\mathrm{Cu})$, ferro $(\mathrm{Fe})$, manganês $(\mathrm{Mn})$ e zinco (Zn) seus valores chegaram a zero à medida que aumentou as concentrações de água residuária.

Tabela 1. Análise dos macro, micronutrientes e sódio $\mathrm{mg} \mathrm{dm}^{-3}$ na água residuária da piscicultura, após retirada da água no reservatório.

\begin{tabular}{lllllllllll}
\hline \multirow{2}{*}{ Protoc. } & \multirow{2}{*}{ Tratamento } & \multicolumn{8}{c}{$\mathbf{~ m g} / \mathbf{d m}^{\mathbf{3}}$} \\
\cline { 3 - 11 } & $\mathbf{p H}$ & $\mathbf{K}$ & $\mathbf{N a}$ & $\mathbf{C a}$ & $\mathbf{M g}$ & $\mathbf{C u}$ & $\mathbf{F e}$ & $\mathbf{M n}$ & $\mathbf{Z n}$ \\
\hline 1 & Amostra 0\% & 6,24 & 2,10 & 3,50 & 1,57 & 1,38 & 0,08 & 0,00 & 0,02 & 0,03 \\
2 & Amostra 25\% & 8,51 & 2,40 & 3,90 & 1,48 & 1,40 & 0,00 & 0,00 & 0,00 & 0,00 \\
3 & Amostra 50\% & 9,84 & 2,70 & 3,90 & 1,30 & 1,40 & 0,00 & 0,00 & 0,00 & 0,00 \\
4 & Amostra 75\% & 9,97 & 2,00 & 4,10 & 1,21 & 1,43 & 0,00 & 0,00 & 0,00 & 0,00 \\
5 & Amostra 100\% & 10,55 & 3,50 & 4,20 & 1,13 & 1,28 & 0,00 & 0,00 & 0,00 & 0,00 \\
6 & Amostra 125\% & 6,36 & 2,00 & 2,40 & 0,06 & 1,65 & 0,00 & 0,00 & 0,00 & 0,00 \\
\hline
\end{tabular}


Os micronutrientes apresentaram um grau de significância mínima, apesar de serem necessários para às plantas, mesmo requerido em pequenas quantidades em relação ao nitrogênio, por exemplo, torna-se necessária à sua produtividade e seu desenvolvimento vegetativo. $\mathrm{O}$ fornecimento desses micronutrientes à planta se dá de várias formas, pode ser na adubação de fundação, depois de implantado da cultura, através da adubação foliar, e, ainda, aplicando diretamente na semente (LUCHESE et al.; 2004).

A concentração de $125 \%$ em relação no $\mathrm{K}$ e $\mathrm{Na}$ apresentaram diferença havendo influência significativa dos tratamentos aplicados na concentração deste nutriente nas folhas. Isto porque, o potássio é um dos nutrientes contidos em maior proporção nas águas de concentração.

O Mn, não houve diferença significativa entre as concentrações de água residuária, sendo os valores inferiores em relação à testemunha. De acordo com Pavan et al. (1982), a toxidez do manganês é um dos principais fatores que prejudicam o crescimento das plantas; o excesso deste micronutriente geralmente afeta mais severamente a parte aérea do que as raízes e, aparentemente, as plantas absorvem e transportam o manganês em quantidades excessivas, o que resulta em acúmulo nas folhas.

Os resultados da variável altura de plantas são apresentados na tabela 2. Para esta variável, os resultados foram significativamente diferentes para as coletas realizadas aos 5, 45 e 50 (DAG). Entretanto, para as demais avaliações não houve diferença significativa nesse parâmetro. Aos 5 DAG a maior altura das plantas foi observada na concentração de $100 \%$, apresentado altura de $2,37 \mathrm{~cm}$, enquanto que as plantas sem tratamento com água residuária obtiveram altura média de $1,71 \mathrm{~cm}$. As mudas de pimentão não tratadas com água residuária (0\%), aos 45 e 50 DAG, também atingiram menores alturas em comparação com os demais tratamentos (Tabela 2).

Tabela 2. Altura das plantas (AP) em $\mathrm{cm}$ (média $\pm \mathrm{EP}^{1}$ ) de pimentão, submetidas a diferentes concentrações de água residuária de piscicultura, até os 50 DAG.

\begin{tabular}{|c|c|c|c|c|c|c|c|c|c|}
\hline \multirow[b]{2}{*}{ (DAG) } & \multicolumn{6}{|c|}{ Concentrações (\%) } & \multirow[b]{2}{*}{$\mathrm{F}$} & \multirow[b]{2}{*}{$P$} & \multirow[b]{2}{*}{ CV } \\
\hline & 0 & 25 & 50 & 75 & 100 & 125 & & & \\
\hline \multirow{2}{*}{5} & $1,71 \pm$ & $1,83 \pm$ & $2,24 \pm$ & $2,36 \pm$ & $2,37 \pm$ & $2,08 \pm$ & \multirow{2}{*}{5,51} & 0,0 & \multirow{2}{*}{11,22} \\
\hline & $0,06 \mathrm{c}$ & 0,15 bc & $0,12 \mathrm{ab}$ & $0,15 a b$ & $0,11 \mathrm{a}$ & $0,05 \mathrm{abc}$ & & 2 & \\
\hline \multirow{2}{*}{10} & $2,35 \pm$ & $2,49 \pm$ & $2,65 \pm$ & $2,60 \pm$ & $2,62 \pm$ & $2,27 \pm$ & \multirow{2}{*}{ 2,95 } & 0,0 & \multirow{2}{*}{7,25} \\
\hline & $0,06 \mathrm{a}$ & 0,06 a & $0,00 \mathrm{a}$ & 0,15 a & $0,12 \mathrm{a}$ & $0,04 \mathrm{a}$ & & 6 & \\
\hline \multirow{2}{*}{15} & $3,33 \pm$ & $3,44 \pm$ & $3,77 \pm$ & $3,51 \pm$ & $3,79 \pm$ & $3,58 \pm$ & \multirow{2}{*}{1,66} & 0,1 & \multirow{2}{*}{7,90} \\
\hline & $0,14 \mathrm{a}$ & $0,14 \mathrm{a}$ & 0,03 a & $0,18 \mathrm{a}$ & $0,13 \mathrm{a}$ & $0,15 \mathrm{a}$ & & 9 & \\
\hline \multirow{2}{*}{20} & $4,03 \pm$ & $4,25 \pm$ & $4,69 \pm$ & $4,72 \pm$ & $4,68 \pm$ & $4,65 \pm$ & \multirow{2}{*}{1,79} & 0,1 & \multirow{2}{*}{9,64} \\
\hline & $0,42 \mathrm{a}$ & $0,02 \mathrm{a}$ & $0,11 \mathrm{a}$ & $0,12 \mathrm{a}$ & $0,14 \mathrm{a}$ & $0,21 \mathrm{a}$ & & 6 & \\
\hline \multirow{2}{*}{25} & $4,30 \pm$ & $4,66 \pm$ & $5,16 \pm$ & $5,12 \pm$ & $5,27 \pm$ & $5,16 \pm$ & \multirow{2}{*}{2,91} & 0,0 & \multirow{2}{*}{8,99} \\
\hline & 0,39 a & $0,02 \mathrm{a}$ & 0,13 a & 0,16 a & $0,23 \mathrm{a}$ & 0,19 a & & 6 & \\
\hline \multirow{2}{*}{30} & $4,60 \pm$ & $4,97 \pm$ & $5,48 \pm$ & $5,32 \pm$ & $5,40 \pm$ & $5,51 \pm$ & \multirow{2}{*}{1,99} & 0,1 & \multirow{2}{*}{9,75} \\
\hline & $0,47 \mathrm{a}$ & $0,12 \mathrm{a}$ & $0,14 \mathrm{a}$ & $0,17 \mathrm{a}$ & $0,19 \mathrm{a}$ & $0,23 \mathrm{a}$ & & 2 & \\
\hline \multirow{2}{*}{35} & $4,75 \pm$ & $4,81 \pm$ & $5,65 \pm$ & $5,55 \pm$ & $5,52 \pm$ & $5,87 \pm$ & \multirow{2}{*}{2,28} & 0,0 & \multirow{2}{*}{11,44} \\
\hline & $0,54 \mathrm{a}$ & $0,02 \mathrm{a}$ & 0,18 a & 0,26 a & $0,31 \mathrm{a}$ & $0,25 \mathrm{a}$ & & 8 & \\
\hline \multirow{2}{*}{40} & $4,85 \pm$ & $4,92 \pm$ & $5,83 \pm$ & $5,58 \pm$ & $5,69 \pm$ & $6,03 \pm$ & \multirow{2}{*}{2,89} & 0,0 & \multirow{2}{*}{10,40} \\
\hline & $0,51 \mathrm{a}$ & $0,04 \mathrm{a}$ & 0,18 a & 0,17 a & $0,31 \mathrm{a}$ & $0,25 \mathrm{a}$ & & 6 & \\
\hline \multirow[t]{2}{*}{45} & 4,92 \pm & $5,05 \pm$ & $5,82 \pm$ & $5,83 \pm$ & $6,01 \pm$ & $6,34 \pm$ & \multirow{2}{*}{3,79} & 0,0 & \multirow{2}{*}{10,08} \\
\hline & 0,52 b & $0,03 \mathrm{~b}$ & $0,21 \mathrm{ab}$ & $0,19 a b$ & $0,29 \mathrm{ab}$ & $0,21 \mathrm{a}$ & & 1 & \\
\hline \multirow[t]{2}{*}{50} & $5,04 \pm$ & $5,35 \pm$ & $6,14 \pm$ & $5,66 \pm$ & $6,13 \pm$ & $6,55 \pm$ & 3,61 & 0,0 & 10,15 \\
\hline & $0,51 \mathrm{~b}$ & $0,03 \mathrm{ab}$ & $0,24 \mathrm{ab}$ & $0,22 \mathrm{ab}$ & $0,32 \mathrm{ab}$ & $0,28 \mathrm{a}$ & & 1 & \\
\hline
\end{tabular}

${ }^{1}$ Médias seguidas pela mesma letra, dentro de cada linha, não diferem entre si ao nível de 5\% de probabilidade pelo teste Tukey. 


\section{PRODUÇÃO DE MUDAS DE PIMENTÃO IRRIGADAS COM EFLUENTES DE PISCICULTURA}

De acordo com Mota et al. (1998) em pesquisa realizada com irrigação com água residuária em sorgo, a média da altura das plantas irrigadas com água tratada foi menor do que as irrigadas com água residuária, os maiores resultados em altura de plantas foram obtidos no tratamento com $100 \%$ de água residuária, sendo o melhor resultado em comparação as demais concentrações. Já, segundo Nascimento et al. (2004), estudando irrigação de mamoneira com água de abastecimento e água residuária, verificaram incrementos na altura de plantas e no diâmetro caulinar. Ferreira et al. (2005), em estudos com algodoeiro herbáceo verificaram que a água residuária promoveu maior crescimento de plantas (altura e área foliar por planta) em relação a água de abastecimento.

A variável AP de pimentão, em função das diferentes concentrações de água residuária, seguiu comportamento cúbico de regressão ( $F=42,46 ; P=0,00)$, conforme pode ser observado na figura 1 . A taxa de crescimento das mudas submetidas a $125 \%$ de água residuária foram $58 \%$ superiores em comparação com aquelas submetidas a $0 \%$.

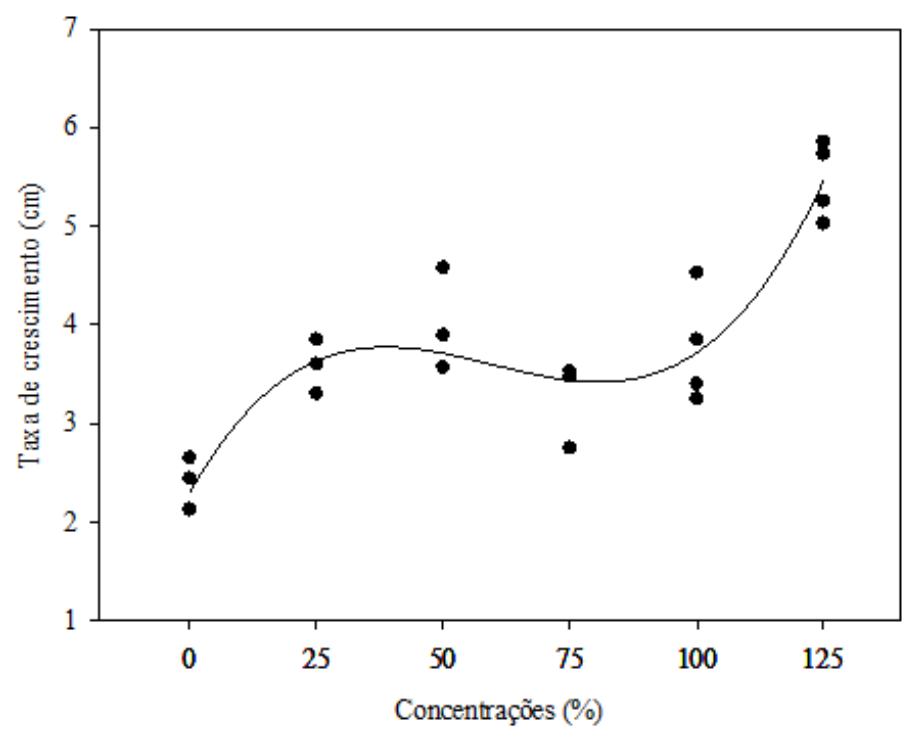

Figura 1. Análise de regressão da variável altura de plantas (AP) em $\mathrm{cm}$, de mudas de pimentão submetidas a diferentes concentrações de água residuária de piscicultura, até os 50 dias após a germinação, $\left(R^{2}=86,43 ; y=\right.$ $\left.0,1186+0,4874 x-0,1533 x^{2}+0,7509 x^{3}\right)$.

A AP de pimentão, também apresentou comportamento cúbico de regressão para as concentrações de $0 \quad(\mathrm{~F}=$ 36,03; $P=0,00), 25 \quad(\mathrm{~F}=440,64 ; P=0,00)$, $50 \quad(\mathrm{~F}=119,00 ; P=0,00), \quad 75 \quad(\mathrm{~F}=136,39 ;$ $P=0,00), \quad 100 \quad(\mathrm{~F}=104,26 ; P=0,00)$ e $125 \%(\mathrm{~F}=230,09 ; P=0,00) \quad$ (Figura 2). Em ordem decrescente, as concentrações de água residuária que originaram maiores alturas de plantas de pimentão ao final da avaliação (50 DAG) foram as de 125,
50, 75, 100, 25 e $0 \%$, com valores finais de altura de 7,64; 7,23; 7,11; 7,03; 6,45 e $5,92 \mathrm{~cm}$, respectivamente (Figura 2).

Augusto et al. (2007) reportaram que embora as mudas produzidas com essa água residuária tenham apresentado crescimento inferior àquelas produzidas com fertilizantes minerais, não foram constatadas mortalidade, deficiência ou toxidez aparente. 

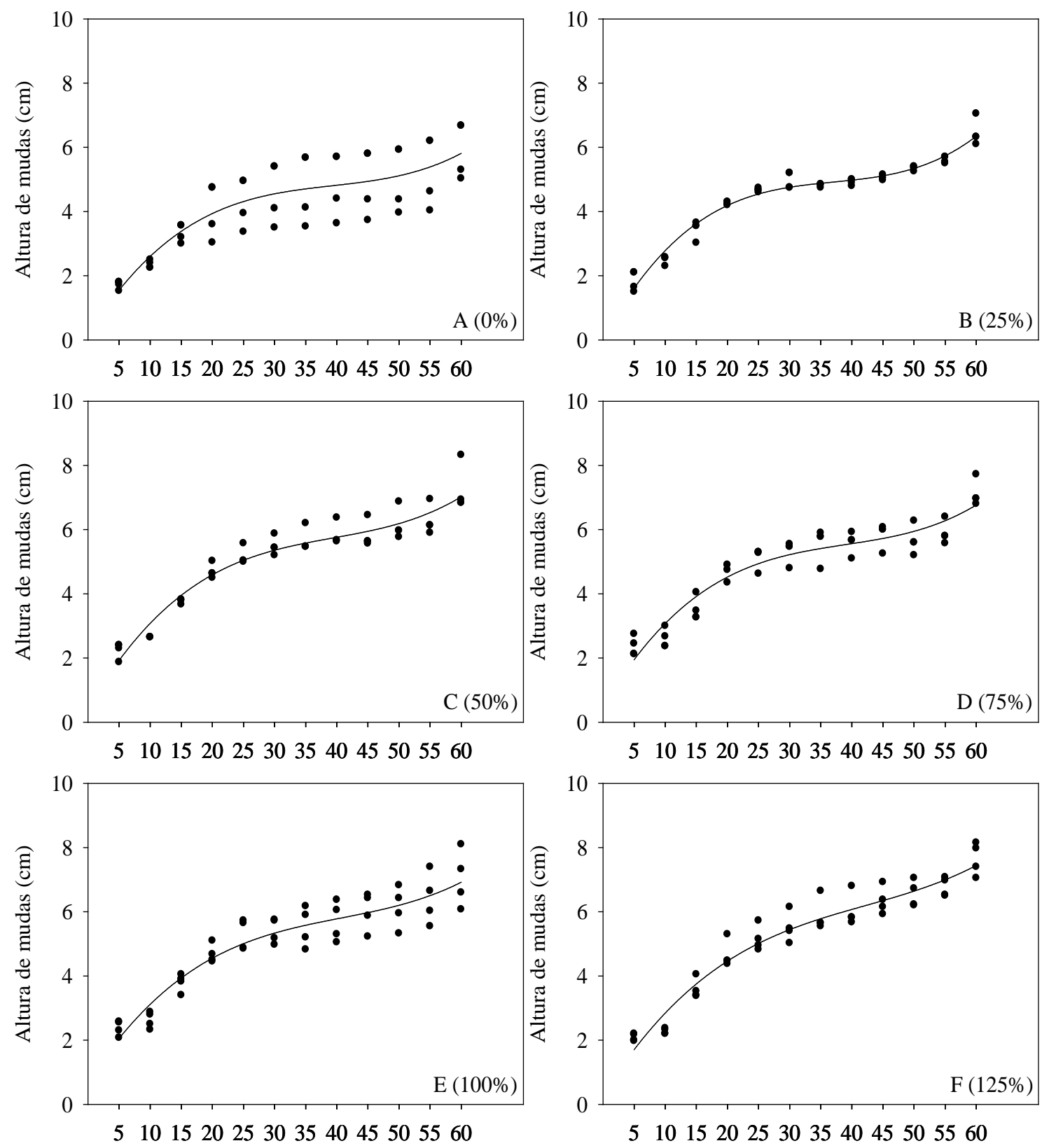

Dias após a germinação

Dias após a germinação

Figura 2. Análise de regressão da altura das plantas (AP) em cm de mudas de pimentão, submetidas a diferentes concentrações (figuras A a F) de água residuária de piscicultura até os 50 DAG. Fig.2A $\left(R^{2}=71,07 ; y=\right.$ $\left.0,1704+0,3102 x-0,7346 x^{2}+0,6235 x^{3}\right)$. Fig. $2 B\left(R^{2}=96,78 ; y=0,4532+0,3564 x-0,9105 x^{2}+0,8190 x^{3}\right)$. Fig. $2 C\left(R^{2}=\right.$ 93,14; $\left.y=0,4347+0,3337 x-0,7579 x^{2}+0,6408 x^{3}\right)$. Fig.2D $\quad\left(R^{2}=\quad 90,29 ; \quad y=0,4813+0,3296 x-\right.$ $\left.0,7701 x^{2}+0,6586 x^{3}\right)$.Fig. $2 E \quad\left(R^{2}=87,67 ; y=0,7050+0,3001 x-0,6442 x^{2}+0,5278 x^{3}\right) \cdot$ Fig. $2 F \quad\left(R^{2}=94,02 ; y=\right.$ $\left.0,2972+0,3104 x-0,6075 x^{2}+0,4810 x^{3}\right)$.

Os dados da variável largura das folhas (LF) estão apresentados na tabela 3. significativas entre as concentrações de água Os resultados não apresentaram diferenças

Tabela 3. Largura média das folhas (LF) em $\mathrm{cm}$ (média $\pm \mathrm{EP}^{1}$ ) de pimentão, submetidas a diferentes concentrações de água residuária de piscicultura, até os 50 DAG. 


\begin{tabular}{|c|c|c|c|c|c|c|c|c|c|}
\hline \multirow[b]{2}{*}{ (DAG) } & \multicolumn{6}{|c|}{ Concentrações (\%) } & \multirow[b]{2}{*}{$\mathrm{F}$} & \multirow[b]{2}{*}{$P$} & \multirow[b]{2}{*}{$\mathrm{CV}$} \\
\hline & 0 & 25 & 50 & 75 & 100 & 125 & & & \\
\hline \multirow{2}{*}{5} & $0,55 \pm$ & $0,63 \pm$ & $0,61 \pm$ & $0,62 \pm$ & $0,60 \pm$ & $0,57 \pm$ & \multirow{2}{*}{1,25} & \multirow{2}{*}{0,32} & \multirow{2}{*}{8,75} \\
\hline & $0,04 \mathrm{a}$ & $0,02 \mathrm{a}$ & $0,01 \mathrm{a}$ & $0,01 \mathrm{a}$ & $0,01 \mathrm{a}$ & $0,03 \mathrm{a}$ & & & \\
\hline \multirow{2}{*}{10} & $0,87 \pm$ & $0,76 \pm$ & $0,78 \pm$ & $0,77 \pm$ & $0,77 \pm$ & $0,78 \pm$ & \multirow{2}{*}{0,99} & $>$ & \multirow{2}{*}{10,11} \\
\hline & $0,07 \mathrm{a}$ & $0,02 \mathrm{a}$ & $0,01 \mathrm{a}$ & $0,03 \mathrm{a}$ & $0,02 \mathrm{a}$ & $0,04 \mathrm{a}$ & & 0,05 & \\
\hline \multirow{2}{*}{15} & $1,36 \pm$ & $1,45 \pm$ & $1,53 \pm$ & $1,41 \pm$ & $1,37 \pm$ & $2,74 \pm$ & \multirow{2}{*}{1,10} & \multirow{2}{*}{0,39} & \multirow{2}{*}{62,58} \\
\hline & $0,10 \mathrm{a}$ & $0,03 \mathrm{a}$ & $0,11 \mathrm{a}$ & 0,07 a & $0,07 \mathrm{a}$ & $1,28 \mathrm{a}$ & & & \\
\hline \multirow{2}{*}{20} & $1,58 \pm$ & $1,90 \pm$ & $1,77 \pm$ & $1,65 \pm$ & $1,76 \pm$ & $1,86 \pm$ & \multirow{2}{*}{1,44} & \multirow{2}{*}{0,25} & \multirow{2}{*}{11,57} \\
\hline & 0,10 a & $0,14 \mathrm{a}$ & $0,07 \mathrm{a}$ & $0,05 \mathrm{a}$ & 0,09 a & $0,11 \mathrm{a}$ & & & \\
\hline \multirow{2}{*}{25} & $1,61 \pm$ & $1,96 \pm$ & $1,84 \pm$ & $1,90 \pm$ & $1,85 \pm$ & $2,00 \pm$ & \multirow{2}{*}{2,02} & \multirow{2}{*}{0,12} & \multirow{2}{*}{10,14} \\
\hline & $0,11 \mathrm{a}$ & 0,13 a & $0,05 \mathrm{a}$ & $0,05 \mathrm{a}$ & 0,07 a & $0,10 \mathrm{a}$ & & & \\
\hline \multirow{2}{*}{30} & $1,67 \pm$ & $1,95 \pm$ & $1,92 \pm$ & $2,00 \pm$ & $1,95 \pm$ & $2,08 \pm$ & \multirow{2}{*}{2,25} & \multirow{2}{*}{0,09} & \multirow{2}{*}{9,44} \\
\hline & 0,10 a & $0,14 \mathrm{a}$ & $0,06 \mathrm{a}$ & $0,03 \mathrm{a}$ & $0,04 \mathrm{a}$ & 0,09 a & & & \\
\hline \multirow{2}{*}{35} & $1,80 \pm$ & $2,08 \pm$ & $1,93 \pm$ & $2,08 \pm$ & $2,10 \pm$ & $2,16 \pm$ & \multirow{2}{*}{1,32} & \multirow{2}{*}{0,29} & \multirow{2}{*}{10,84} \\
\hline & 0,09 a & 0,18 a & $0,07 \mathrm{a}$ & $0,04 \mathrm{a}$ & $0,08 \mathrm{a}$ & $0,11 \mathrm{a}$ & & & \\
\hline \multirow{2}{*}{40} & $1,92 \pm$ & $2,15 \pm$ & $2,08 \pm$ & $2,20 \pm$ & $2,26 \pm$ & $2,23 \pm$ & 1.35 & 0.28 & 9,95 \\
\hline & $0,08 \mathrm{a}$ & 0,15 a & $0,12 \mathrm{a}$ & $0,03 \mathrm{a}$ & 0,07 a & $0,12 \mathrm{a}$ & כב, & & \\
\hline 45 & $2,04 \pm$ & $2,32 \pm$ & $2,56 \pm$ & $2,30 \pm$ & $2,38 \pm$ & $2,30 \pm$ & 1,07 & 0,40 & 13,95 \\
\hline 4 & 0,06 a & 0,18 a & $0,30 \mathrm{a}$ & 0,05 a & 0,09 a & 0,12 a & & $0,+0$ & \\
\hline 50 & $2,18 \pm$ & $2,46 \pm$ & $2,26 \pm$ & $2,45 \pm$ & $2,50 \pm$ & $2,40 \pm$ & 1,38 & 0,27 & 9,12 \\
\hline & $0,06 \mathrm{a}$ & $0,18 \mathrm{a}$ & $0,08 \mathrm{a}$ & $0,05 \mathrm{a}$ & $0,09 \mathrm{a}$ & $0,11 \mathrm{a}$ & & & \\
\hline
\end{tabular}

${ }^{1}$ Médias seguidas pela mesma letra, dentro de cada linha, não diferem entre si ao nível de 5\% de probabilidade pelo teste Tukey.

A largura de folhas das mudas de pimentão também seguiu um comportamento cúbico de regressão nas concentrações de água residuária de $0(\mathrm{~F}=165,42 ; P=0,00), 25(\mathrm{~F}=$ 76,09; $P=0,00), 50(\mathrm{~F}=80,56 ; P=0,00), 75$ $(\mathrm{F}=480,96 ; P=0,00), 100 \quad(\mathrm{~F}=265,90 ; P=$ $0,00)$ e $125 \%(F=129,34 ; P=0,00)$ conforme é mostrado na figura 3 . $\mathrm{O}$ maior valor comparativo para a variável LF entre os 50 e 5 DAG, foi observado para a concentração de $125 \%$, com taxa de crescimento em cerca de $80 \%$ a mais aos 50 DAG em comparação com os 5 DAG. Para as demais concentrações, a taxa de crescimento foi de $76 \%$ (na concentração 50\%), 78\% (nas concentrações 0, 25 e 75\%) e 79\% (na concentração 100\%).

Chaves e Guerra (2006), Alcântara (2003), utilizando lodo de esgoto como fonte de nutrientes para o algodão herbáceo, encontrou melhores resultados em área foliar por planta quando comparado com a testemunha absoluta e com o tratamento contendo adubo mineral. 

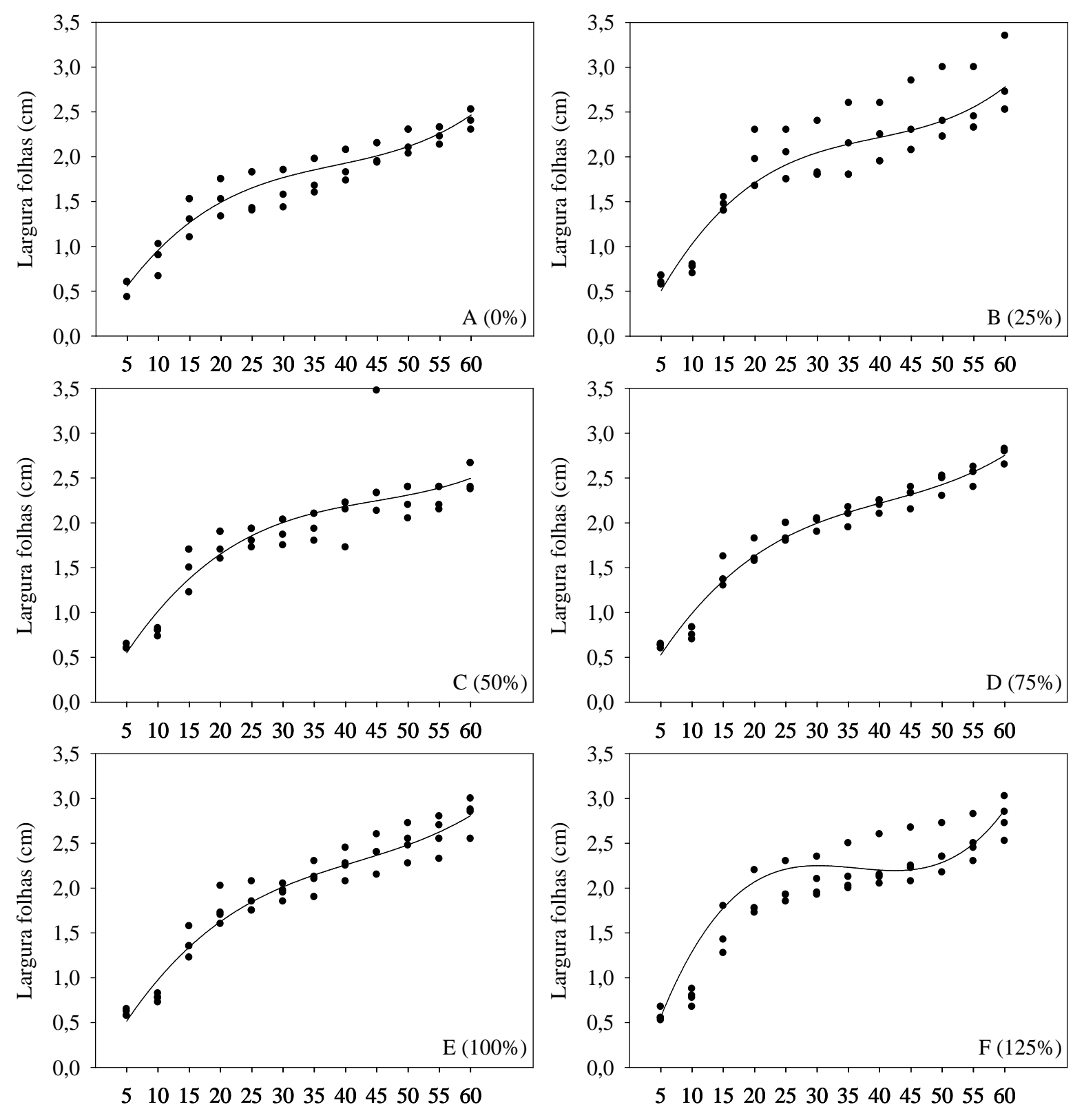

Dias após a germinação

Dias após a germinação

Figura 3. Análise de regressão da largura de folhas (LF) em cm, de mudas de pimentão, submetidas a diferentes concentrações de água residuária de piscicultura (Figuras A a F), até os 50 DAG. Fig.2A $\left(R^{2}=91,86\right.$; $\left.y=0,3497+0,1169 x-0,2670 x^{2}+0,2327 x^{3}\right)$. Fig. $2 B \quad\left(R^{2}=83,84 ; y=-0,1686+0,1524 x-0,3515 x^{2}+0,2990 x^{3}\right)$. Fig.2C $\left(R^{2}=84,60 ; \quad y=-0,2718+0,1280 x-0,2590 x^{2}+0,1928 x^{3}\right) . \quad$ Fig.2D $\quad\left(R^{2}=97,04 ; \quad y=\quad-0,5410+0,1289 x-\right.$ $\left.0,2673 x^{2}+0,2173 x^{3}\right)$. Fig. $2 E\left(R^{2}=94,77 ; y=-0,511+0,1263 x-0,2516 x^{2}+0,2010 x^{3}\right)$. Fig. $2 F\left(R^{2}=73,67 ; y=\right.$ $\left.0,4551+0,3759 x-0,2091 x^{2}+0,1689 x^{3}\right)$.

Os dados de número de folhas verdadeiras (NF) estão apresentados na tabela 4. O NF sofreu influência do percentual de concentração de água residuária, apenas, aos 15, 25, 30, 35, 40, 45 e 50 DAG. Na concentração de $50 \%$ de diluição de água residuária o número médio de folhas verdadeiras foi maior para os 15, 25 e 35 DAG. Entretanto, aos 30, 35, 40, 45 e 50 DAG, as mudas submetidas a uma concentração de $100 \%$ originaram maior número médio de folhas verdadeiras em comparação com as demais concentrações avaliadas. 
Tabela 4. Número de folhas verdadeiras (NF) (média $\pm \mathrm{EP}^{1}$ ) de pimentão, submetidas a diferentes concentrações de água residuária de piscicultura até os 50 DAG.

\begin{tabular}{|c|c|c|c|c|c|c|c|c|c|}
\hline \multirow[b]{2}{*}{ (DAG) } & \multicolumn{6}{|c|}{ Concentrações (\%) } & \multirow[b]{2}{*}{$F$} & \multirow[b]{2}{*}{$P$} & \multirow[b]{2}{*}{ CV } \\
\hline & 0 & 25 & 50 & 75 & 100 & 125 & & & \\
\hline 5 & $\begin{array}{l}2,00 \pm \\
0,00 \mathrm{a}\end{array}$ & $\begin{array}{l}2,00 \pm \\
0,00 \mathrm{a}\end{array}$ & $\begin{array}{l}2,00 \pm \\
0,00 \mathrm{a}\end{array}$ & $\begin{array}{l}2,12 \pm \\
0,07 \mathrm{a}\end{array}$ & $\begin{array}{l}2,06 \pm \\
0,06 \mathrm{a}\end{array}$ & $\begin{array}{l}2,00 \pm \\
0,00 \mathrm{a}\end{array}$ & 1,80 & 0,16 & 3,83 \\
\hline 10 & $\begin{array}{l}3,87 \pm \\
0,37 \mathrm{a}\end{array}$ & $\begin{array}{l}3,87 \pm \\
0,12 \mathrm{a}\end{array}$ & $\begin{array}{l}3,66 \pm \\
0,19 \mathrm{a}\end{array}$ & $\begin{array}{l}3,31 \pm \\
0,23 \mathrm{a}\end{array}$ & $\begin{array}{l}3,81 \pm \\
0,18 \mathrm{a}\end{array}$ & $\begin{array}{l}3,62 \pm \\
0,16 \mathrm{a}\end{array}$ & 1,49 & 0,24 & 9,51 \\
\hline 15 & $\begin{array}{r}5,66 \pm \\
0,37 \mathrm{ab}\end{array}$ & $\begin{array}{c}5,43 \pm \\
0,35 \mathrm{ab}\end{array}$ & $\begin{array}{l}6,00 \pm \\
0,00 \mathrm{a}\end{array}$ & $\begin{array}{c}4,83 \pm \\
0,14 \mathrm{ab}\end{array}$ & $\begin{array}{c}5,18 \pm \\
0,25 \mathrm{ab}\end{array}$ & $\begin{array}{l}4,50 \pm \\
0,22 \mathrm{~b}\end{array}$ & 4,42 & 0,00 & 9,91 \\
\hline 20 & $\begin{array}{l}5,91 \pm \\
0,43 \mathrm{a}\end{array}$ & $\begin{array}{l}5,81 \pm \\
0,18 \mathrm{a}\end{array}$ & $\begin{array}{l}6,33 \pm \\
0,19 \mathrm{a}\end{array}$ & $\begin{array}{l}5,43 \pm \\
0,25 \mathrm{a}\end{array}$ & $\begin{array}{r}5,68 \pm \\
0,44 \mathrm{a}\end{array}$ & $\begin{array}{c}5,56 \pm \\
0,21 \mathrm{a}\end{array}$ & 1,04 & 0,42 & 10,67 \\
\hline 25 & $\begin{array}{l}6,27 \pm \\
0,42 \mathrm{ab}\end{array}$ & $\begin{array}{c}5,87 \pm \\
0,21 \mathrm{ab}\end{array}$ & $\begin{array}{l}6,72 \pm \\
0,19 \mathrm{a}\end{array}$ & $\begin{array}{l}5,14 \pm \\
0,31 \mathrm{~b}\end{array}$ & $\begin{array}{r}6,18 \pm \\
0,37 \mathrm{ab}\end{array}$ & $\begin{array}{c}6,43 \pm \\
0,15 \mathrm{ab}\end{array}$ & 3,40 & 0,02 & 9,7 \\
\hline 30 & $\begin{array}{c}6,00 \pm \\
0,44 \mathrm{ab}\end{array}$ & $\begin{array}{c}5,62 \pm \\
0,21 \mathrm{ab}\end{array}$ & $\begin{array}{c}5,95 \pm \\
0,19 \mathrm{ab}\end{array}$ & $\begin{array}{l}4,79 \pm \\
0,28 b\end{array}$ & $\begin{array}{l}6,25 \pm \\
0,30 \mathrm{a}\end{array}$ & $\begin{array}{l}6,25 \pm \\
0,36 \mathrm{a}\end{array}$ & 3,06 & 0,03 & 10,81 \\
\hline 35 & $\begin{array}{c}4,91 \pm \\
0,08 \mathrm{ab}\end{array}$ & $\begin{array}{c}4,75 \pm \\
0,25 \mathrm{ab}\end{array}$ & $\begin{array}{l}5,20 \pm \\
0,12 \mathrm{a}\end{array}$ & $\begin{array}{l}4,04 \pm \\
0,45 b\end{array}$ & $\begin{array}{l}5,68 \pm \\
0,25 \mathrm{a}\end{array}$ & $\begin{array}{l}5,37 \pm \\
0,16 \mathrm{a}\end{array}$ & 5,10 & 0,00 & 10,15 \\
\hline 40 & $\begin{array}{c}5,29 \pm \\
0,20 \mathrm{ab}\end{array}$ & $\begin{array}{c}4,81 \pm \\
0,23 \mathrm{ab}\end{array}$ & $\begin{array}{c}5,66 \pm \\
0,13 \mathrm{ab}\end{array}$ & $\begin{array}{l}4,56 \pm \\
0,38 \mathrm{~b}\end{array}$ & $\begin{array}{l}5,87 \pm \\
0,38 \mathrm{a}\end{array}$ & $\begin{array}{l}6,00 \pm \\
0,22 \mathrm{a}\end{array}$ & 4,77 & 0,00 & 9,96 \\
\hline 45 & $\begin{array}{l}4,97 \pm \\
0,15 \mathrm{ab}\end{array}$ & $\begin{array}{c}4,31 \pm \\
0,18 \mathrm{bc}\end{array}$ & $\begin{array}{c}4,70 \pm \\
0,27 \mathrm{abc}\end{array}$ & $\begin{array}{l}4,10 \pm \\
0,47 \mathrm{c}\end{array}$ & $\begin{array}{l}5,62 \pm \\
0,36 \mathrm{a}\end{array}$ & $\begin{array}{c}5,43 \pm \\
0,15 \mathrm{ab}\end{array}$ & 4,28 & 0,00 & 12,02 \\
\hline 50 & $\begin{array}{l}4,72 \pm \\
0,17 \mathrm{ab}\end{array}$ & $\begin{array}{l}4,31 \pm \\
0,18 \mathrm{~b}\end{array}$ & $\begin{array}{c}4,54 \pm \\
0,35 \mathrm{ab}\end{array}$ & $\begin{array}{r}4,43 \pm \\
0,29 \mathrm{ab}\end{array}$ & $\begin{array}{l}5,56 \pm \\
0,41 \mathrm{a}\end{array}$ & $\begin{array}{c}5,06 \pm \\
0,06 \mathrm{ab}\end{array}$ & 2,86 & 0,04 & 11,54 \\
\hline
\end{tabular}

${ }^{1}$ Médias seguidas pela mesma letra, dentro de cada linha, não diferem entre si ao nível de 5\% de probabilidade pelo teste Tukey.

Segundo dados de Paiva et al. (2012) o número de folhas (NF) e altura das plantas (AP) apresentaram resultados significativos para mudas de pimentão irrigadas com água residuária. O NF apresentou valores 176\% maiores se comparados aos resultados obtidos com o tratamento com $0 \%$ de água residuária. Já a variável AP obtive resultados superiores em 108,6\%, maiores que o tratamento sem adição de água residuária.

Os resultados das variáveis peso de matéria fresca do sistema radicular (PMFSR), peso de matéria fresca da parte aérea (PMFPA), e peso de matéria seca do sistema radicular (PMSSR) e peso de matéria seca da parte aérea (PMSPA) são apresentados na tabela 5

Tabela 5. Peso de matéria fresca do sistema radicular (PMFSR), peso de matéria fresca da parte aérea (PMFPA), peso de matéria seca do sistema radicular (PMSSR) e peso de matéria seca da parte aérea (PMSPA) em g, (média $\pm \mathrm{EP}^{1}$ ) de mudas de pimentão, submetidas a diferentes concentrações de água residuária de piscicultura, durante o período pós-germinativo até os 50 DAG.

\begin{tabular}{ccccc}
\hline Conc. (\%) & PMFSR & PMFPA & PMSSR & PMSPA \\
\hline 0 & $0,634 \pm 0,087 \mathrm{~b}$ & $0,764 \pm 0,090 \mathrm{~b}$ & $0,076 \pm 0,011 \mathrm{~b}$ & $0,104 \pm 0,012 \mathrm{c}$ \\
25 & $0,592 \pm 0,040 \mathrm{~b}$ & $0,859 \pm 0,049 \mathrm{~b}$ & $0,074 \pm 0,005 \mathrm{~b}$ & $0,115 \pm 0,007 \mathrm{c}$ \\
50 & $0,648 \pm 0,041 \mathrm{~b}$ & $0,944 \pm 0,064 \mathrm{ab}$ & $0,081 \pm 0,004 \mathrm{~b}$ & $0,119 \pm 0,008 \mathrm{~b}$ \\
75 & $0,642 \pm 0,049 \mathrm{~b}$ & $0,847 \pm 0,125 \mathrm{~b}$ & $0,083 \pm 0,008 \mathrm{~b}$ & $0,120 \pm 0,006 \mathrm{~b}$ \\
100 & $0,685 \pm 0,038 \mathrm{~b}$ & $1,083 \pm 0,092 \mathrm{ab}$ & $0,085 \pm 0,006 \mathrm{~b}$ & $0,138 \pm 0,009 \mathrm{~b}$ \\
125 & $0,926 \pm 0,034 \mathrm{a}$ & $1,222 \pm 0,074 \mathrm{a}$ & $0,120 \pm 0,007 \mathrm{a}$ & $0,161 \pm 0,012 \mathrm{a}$ \\
\hline
\end{tabular}


Nascimento et al.

\begin{tabular}{ccccc}
\hline $\mathrm{F}$ & 5,38 & 3,88 & 4,90 & 4,17 \\
$P$ & 0,00 & 0,00 & 0,00 & 0,00 \\
$\mathrm{CV}$ & 23,85 & 28,66 & 27,85 & 24,78 \\
\hline
\end{tabular}

Para as variáveis PMFPA e PMFSR, os valores foram maiores na concentração de $125 \%$ se comparadas com os demais tratamentos. Isto pode ser explicado pelo maior volume de nutrientes disponível às mudas, que, por sua vez, permite um melhor desenvolvimento do sistema radicular, aumentando a área de absorção de nutrientes, e consequentemente, proporcionando um bom desempenho da planta. Esses resultados vêm ao encontro com os obtidos por Bar-Tal et al. (1990) que estudando a interação entre o volume de raiz e a nutrição de mudas de pimentão, demonstraram que o peso das mudas aumentou à medida que o volume de substrato e a concentração de nutrientes na água de irrigação aumentavam.

Oliveira et al. (2012) obtiveram resultados significativos das variáveis matéria fresca do caule, da raiz e da folha, bem como matéria seca do caule e da folha, no seu experimento com sabiá (Mimosa caesalpinieaefolia Benth) irrigado com água residuária. Também segundo Oliveira et al. (2012b), em seu trabalho com pimenta cambuci (Capsicum baccatum L. varpendulum) e Quiabo (Abelmoschus esculentus L.) verificaram incremento na matéria fresca de ambos quando irrigados com água residuária.

\section{CONCLUSÕES}

Os tratamentos com diferentes concentrações de água residuária de piscicultura propiciaram diferenças significativas nas variáveis, largura das folhas e altura das plantas para a cultura do pimentão, sendo que quanto maior a concentração maior foram os valores dessas variáveis, devido a maior disponibilidade de nutrientes a planta.

A água residuária da piscicultura apresentou como alternativa para reuso do ponto de vista nutricional para as plantas, diminuindo o impacto ambiental negativo dos efluentes desse tipo de criação animal.

\section{REFERÊNCIAS}

ALCANTARA, R. de L. Biossólido como fonte de nutriente para o algodão herbáceo e seu efeito residual no milho. 2003. 177p. Tese (Tese de Doutorado em Recursos Naturais). Universidade Federal de Campina Grande, Campina Grande.

AUGUSTO, D. C. C.; GUERRINI, I. A.; ENGEL, V. L.; ROUSSEAU, G. X. Utilização de águas residuárias provenientes do tratamento Biológico de esgotos domésticos na produção de mudas de Eucalyptus grandis hill. Revista Árvore, Viçosa, v.31, n.4, p.745-751, 2007.

BAR-TAL, A.; BAR-JOSEF, B.; KAFKAFI, U. Pepper transplant response to root volume and nutrition in the nursery. Agronomy Journal, Madison, v.82, p. 989-995, 1990.

CHAVES, L. H. G.; GUERRA, H. O. C. Solos Agrícolas. Campina Grande: EDUFCG, 2006.178p.

FERREIRA, O. E.; BELTRÃO, N. E. M.; KONIG, A. Efeitos da aplicação de água residuária e nitrogênio sobre o crescimento e produção do algodão herbáceo. Revista Brasileira de Oleaginosas e Fibrosas, Campina Grande, v. 9, n. 01/03, p. 893-902, 2005.

FILGUEIRA, F. A. R. Manual de Olericultura: Agrotecnologia moderna na produção e comercialização de hortaliças. 2 ed., Viçosa: UFV, 2004. 402 p.

FILGUEIRA, F. A. R. Novo Manual de olericultura: agrotecnologia moderna na 
produção e comercialização de hortaliças. Viçosa: UFV, 2000. 402 p.

LEONARDO, M.; BROETTO, F.; BÔAS, R.L.V.; ALMEIDA, R.S.; JOSÉ ABRAMO MARCHESE, J.A. Produção de frutos de pimentão em diferentes concentrações Salinas. Irriga, Botucatu, v. 12, n. 1, p. 73-77, 2007.

LUCHESE, A. V.; JUNIOR, A. C. G.; LUCHESE, E. B.; BRACCINI, M. do C. L. Emergência e absorção de cobre por plantas de milho (Zea mays) em resposta ao tratamento de sementes com cobre. Ciência Rural, Santa Maria, v.34, n.6, p.1949-1952, 2004.

MOTA, S.; BEZERRA, F. C.; TOMÉ, L. M.; Avaliação do desempenho de culturas irrigadas com esgoto tratado. In: $19^{\circ}$ Congresso Brasileiro de Engenharia Sanitária e Ambiental. 1998.

NASCIMENTO, M. B. H. et al. Utilização de água residuária e biossólido na cultura da mamona: crescimento e desenvolvimento. In: Congresso brasileiro de mamona, 1., 2004, Campina Grande-PB. Anais, Campina Grande: Embrapa. 1 CD-ROM.

OLIVEIRA, J. F.; ALVES, S. M. C.; BATISTA, R. O.; PAIVA, L. A. L.; LIMA, V. I. A.; COSTA, L. L. B.; Fertirrigação de Mudas de Sabiá com Esgoto Doméstico Terciário; In: WINOTEC, 2012.
OLIVEIRA, J. F.; ALVES, S. M. C.; FERREIRA NETO, M.; OLIVEIRA, R. B. Efeito da Água Residuária de Esgoto Doméstico Tratado na Produção de Mudas de Pimenta Cambuci e Quiabo. Revista Enciclopédia Biosfera, Goiania, v.8. $\mathrm{n}^{\mathrm{0}}$ 14, 2012b.

PAIVA. L. A. L.; ALVES, S. M. C.; FERREIRA NETO, M.; OLIVEIRA, R. B.; OLIVEIRA, J. F. Influência da aplicação de esgoto domestico secundário na produção de mudas de pimenta malagueta e pimentão. Centro Cientifico Conhecer. 2012.

PAVAN, M.A.; BINGHAM, F.T.; PRATT, P.F. Toxicity of aluminum to coffee in Ultisols and Oxisols amended with $\mathrm{CaCO} 3$ and $\mathrm{CaSO} 4$. Soil Science Society of America Journal, v.46, p.1201-1207, 1982.

SANDRI, D. Irrigação da cultura da alface com água residuária tratada com leitos cultivados com macrófita. 2003. 186 f. Tese (Doutorado em Engenharia de Água e Solo) Faculdade de Engenharia Agrícola, Universidade Estadual de Campinas, Campinas.

SARIG, S. The immigration for fish culture into general farm irrigation systems in Israel. Bamidgeh 1 (36): 16-20. 1994. 